\title{
Blastic plasmacytoid dendritic cell neoplasm with absolute monocytosis at presentation
}

This article was published in the following Dove Press journal:

Pathology and Laboratory Medicine International

12 February 2015

Number of times this article has been viewed

\author{
Joseph M Jaworski ${ }^{1,2}$ \\ Vanlila K Swami' \\ Rebecca C Heintzelman' \\ Carrie A Cusack ${ }^{3}$ \\ Christina L Chung ${ }^{3}$ \\ Jeremy Peck ${ }^{3}$ \\ Matthew Fanelli ${ }^{3}$ \\ Micheal Styler ${ }^{4}$ \\ Sanaa Rizk ${ }^{4}$ \\ J Steve Hou' \\ 'Department of Pathology and \\ Laboratory Medicine, Hahnemann \\ University Hospital/Drexel University \\ College of Medicine, Philadelphia, \\ PA, USA; '2Department of Pathology, \\ Mercy Fitzgerald Hospital, Darby, PA, \\ USA; ${ }^{3}$ Department of Dermatology, \\ Hahnemann University Hospital/ \\ Drexel University College of \\ Medicine, Philadelphia, PA, USA; \\ ${ }^{4}$ Department of Hematology/ \\ Oncology, Hahnemann University \\ Hospital/Drexel University College \\ of Medicine, Philadelphia, PA, USA
}

Correspondence: J Steve Hou Hahnemann University Hospital/ Department of Pathology and Laboratory Medicine, Mail Stop 435, 245 N I5th Street, Philadelphia, PA 19102-1192

Tel +l 2I57623753

Email j.hou@drexelmed.edu

\begin{abstract}
Blastic plasmacytoid dendritic cell neoplasm is an uncommon malignancy derived from precursors of plasmacytoid dendritic cells. Nearly all patients present initially with cutaneous manifestations, with many having extracutaneous disease additionally. While response to chemotherapy initially is effective, relapse occurs in most, with a leukemic phase ultimately developing. The prognosis is dismal. While most of the clinical and pathologic features are well described, the association and possible prognostic significance between peripheral blood absolute monocytosis $(>1.0 \mathrm{~K} / \mu \mathrm{L})$ and blastic plasmacytoid dendritic cell neoplasm have not been reported. We report a case of a 68 -year-old man who presented with a rash for 4-5 months. On physical examination, there were multiple, dull-pink, indurated plaques on the trunk and extremities. Complete blood count revealed thrombocytopenia, absolute monocytosis of $1.7 \mathrm{~K} / \mu \mathrm{L}$, and a negative flow cytometry study. Biopsy of an abdominal lesion revealed typical features of blastic plasmacytoid dendritic cell neoplasm. Patients having both hematologic and nonhematologic malignancies have an increased incidence of absolute monocytosis. Recent studies examining Hodgkin and non-Hodgkin lymphoma patients have suggested that this is a negative prognostic factor. The association between blastic plasmacytoid dendritic cell neoplasm and absolute monocytosis has not been described and may, in fact, have prognostic value.
\end{abstract}

Keywords: blastic, plasmacytoid, dendritic, monocytosis

\section{Introduction}

Having undergone multiple different name revisions (eg, blastic natural killer cell lymphoma, agranular CD4+/CD56+ hematodermic neoplasm) due to the initially enigmatic cell of origin, ${ }^{1}$ blastic plasmacytoid dendritic cell neoplasm (BPDCN) is recognized in the current World Health Organization Classification of Tumours of Haematopoietic and Lymphoid Tissues ${ }^{2}$ as an aggressive neoplasm derived from the precursors of plasmacytoid dendritic cells and categorically placed under the heading "Acute myeloid leukemia (AML) and related precursor neoplasms." BPDCN is a rare entity, first described in $1994^{3}$ and with less than 200 cases reported since. ${ }^{4}$ Petrella et $\mathrm{al}^{1}$ and Cota et $\mathrm{al}^{5}$ have both examined relatively large series of patients (30 and 33, respectively) with this uncommon disease with follow-up for most of the patients. The mean age at diagnosis is 66 years (age range, 8-96 years) with a male predominance of 3.5:1., ${ }^{1,5}$ Virtually all patients present with cutaneous lesions that can either be single, multiple, or generalized nodules, plaques, and bruise-like areas. ${ }^{1,2,5}$ However, the rare case has been reported of leukemic presentation without cutaneous involvement. ${ }^{6}$ Of patients who undergo complete staging, 63\% have cutaneous involvement only, while $37 \%$ have either nodal and/or bone marrow involvement in addition to skin disease. ${ }^{1,5}$ 
Of note, these large series do not comment on the presence or absence of peripheral blood absolute monocytosis (PBAM).

Due to its rarity, no standardized treatment modalities exist for BPDCN. Polychemotherapy, radiotherapy, and stem cell transplant have all been used. ${ }^{1}$ The majority $(75 \%-90 \%)$ of patients show an initial response to therapy (regression or disappearance of disease), but relapses occur in most of these patients. ${ }^{1,2}$ The skin is involved in most cases at first relapse, with or without leukemic dissemination. ${ }^{1}$ In one study, the range for time to first relapse from initial treatment was 2-31 months. ${ }^{1}$ Despite treatment, the prognosis for patients with BPDCN is poor, with an average survival of 14 months. ${ }^{1}$ In a few reported cases, long-term remissions have been achieved only with chemotherapy and allogeneic stem cell transplantation. ${ }^{5,7-9}$ Whether or not the disease is limited to the skin at presentation seems not to have an effect on survival. ${ }^{5,8}$ For this reason, some advocate treating patients with BPDCN aggressively, regardless of staging. ${ }^{5,8}$

\section{Material and methods}

Skin punch biopsy, bone marrow aspirate, and core biopsy specimens were obtained and processed according to standard techniques.

\section{Immunohistochemistry}

Immunohistochemical stains were performed on the skin biopsy using an Autostainer (DakoCytomation, Inc., Carpinteria, CA, USA). Sections were deparaffinized in xylene and alcohol. Antigen retrieval for CD4, CD43, CD56, CD68, CD3, CD5, CD8, CD20, PAX-5, CD10, CD30, EMA, ALK-1, CD34, and MPO was accomplished by steaming the paraffin sections in ethylenediaminetetraacetic acid (EDTA) buffer for 30 minutes using a commercial vegetable steamer. Detection was with the avidin-biotin peroxidase complex.

\section{Flow cytometry}

Three-color flow cytometry was performed on the skin biopsy, peripheral blood, and bone marrow aspirate using a FACScan (Becton, Dickinson, and Company, San Jose, CA, USA). Briefly, single-cell suspension of the biopsy, EDTA anticoagulated peripheral blood, or marrow were incubated with antibodies directed to the following antigens: CD2, CD3, CD4, CD8, CD19, CD13, CD14, CD15, CD45, CD64, CD34, HLA-DR and CD117.

\section{Case report}

We report a case of a 68-year-old African American male with diabetes mellitus, hyperlipidemia, and gastroesophageal reflux disease who presented with a rash of 4-5 months duration. According to the patient, the rash began on the chest and subsequently spread to his stomach, back, scalp, face, arms, legs, and genitals. He described the rash as pruritic and nontender, except for a painful raised lesion on his abdomen. The patient denied any fever or recent changes in medications. On physical examination, multiple dull-pink, indurated plaques were present on the trunk and extremities, as well as firm, red, erythematous, nonmobile and tender papules on the abdomen (Figure 1). A cutaneous biopsy was performed on the abdominal lesion. Complete blood count showed a white blood cell count of $8.8 \mathrm{~K} / \mu \mathrm{L}$, hemoglobin of $11.0 \mathrm{~g} / \mathrm{dL}$, and platelet count of $114 \mathrm{~K} / \mu \mathrm{L}$. The differential revealed: neutrophils $57.0 \%$, lymphocytes $23.1 \%$, monocytes $19.5 \%$, basophils $0.3 \%$, and eosinophils $0.1 \%$. The absolute monocyte count was $1.7 \mathrm{~K} / \mu \mathrm{L}$. Flow cytometry of the peripheral blood and bone marrow revealed no immunophenotypically abnormal cell population. Before a pathologic diagnosis was rendered, a preliminary, clinical diagnosis of cutaneous T-cell lymphoma was made and treatment with topical agents and phototherapy was initiated. The patient did have subsequent improvement of the lesions; however, upon evaluation by oncology, due to the aggressiveness of the tumor and possible bone metastasis, a bone marrow transplant was recommended. The patient was resistant to this recommendation and instead opted for induction chemotherapy to induce remission, with hyper CVAD (cyclophosphamide, vincristine, doxorubicin, dexamethasone) four times a month for a duration of 6 months. Patient was lost to follow-up after the initial chemotherapy.

Sections from the skin biopsy revealed dermis infiltrated by a diffuse infiltrate of medium-sized monomorphic cells with irregular nuclear contours, fine and evenly distributed

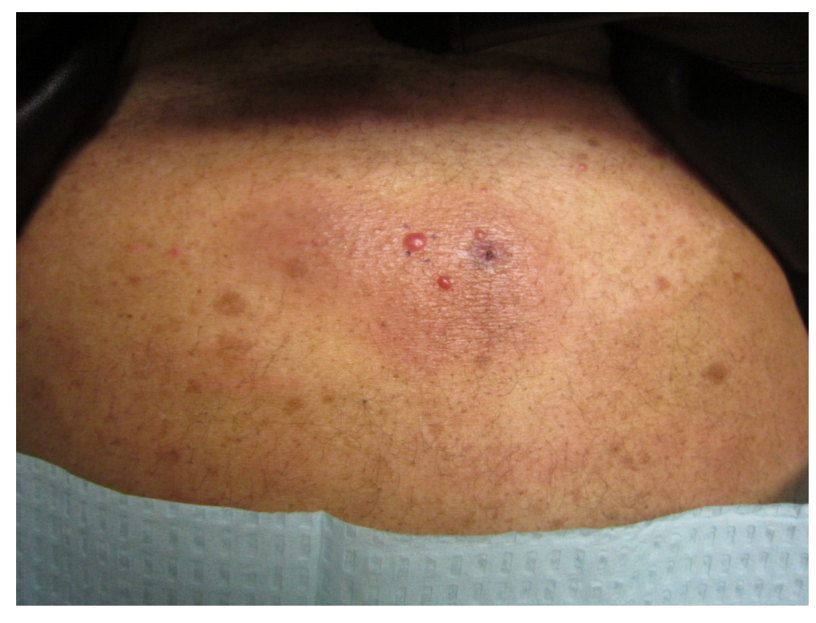

Figure I Abdomen with dull, pink-brown, indurated plaques with several red papules. 
chromatin, and small nucleoli, sometimes multiple (Figures 2 and 3). Angiocentric and angiodestructive lesions were not present. Scattered mitotic figures were seen. The epidermis was spared. An extensive immunohistochemical panel was performed, showing the atypical cells positive for CD4, CD43, CD56, and CD68 (dot-like distribution) (Figure 4). All the following remaining stains were negative: CD3, CD5, CD8, CD20, PAX-5, CD10, MUM-1, BCL-6, CD30, EMA, ALK-1, CD34, MPO and Leder stain. Ki-67 highlighted $50 \%$ of neoplastic cell nuclei. Flow cytometry performed on a subsequent specimen revealed a cell population that coexpressed CD13, HLA-DR, and CD45 (dim), while CD3, CD4, CD8, CD19, CD14, CD15, CD64, CD34, and CD117 were negative. A diagnosis of blastic plasmacytoid dendritic cell neoplasm was rendered based on the above findings.

\section{Discussion}

We present a case of BPDCN without peripheral blood involvement as demonstrated by negative flow cytometry. However, the peripheral blood revealed an absolute

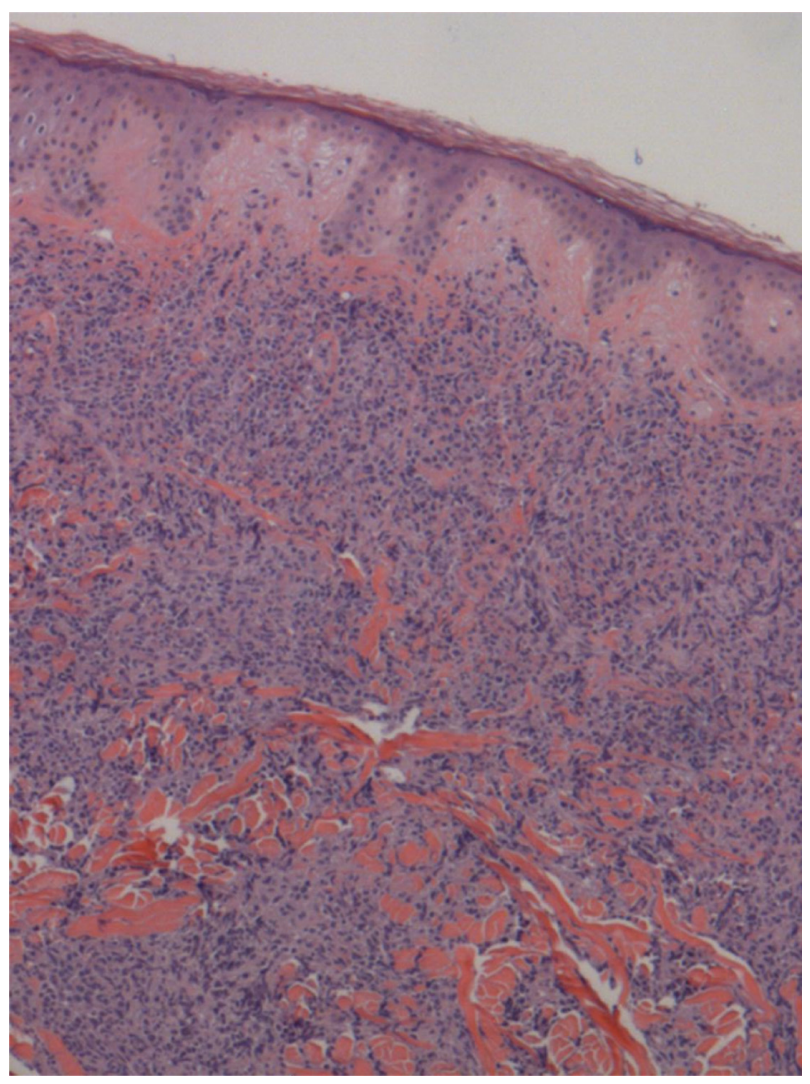

Figure 2 Section of abdominal lesion from skin biopsy.

Notes: Abdomen, magnification $=40 \times$, hematoxylin and eosin staining. Sections through the abdominal lesion show infiltration of the dermis by a monomorphic cell population. The epidermis is spared.

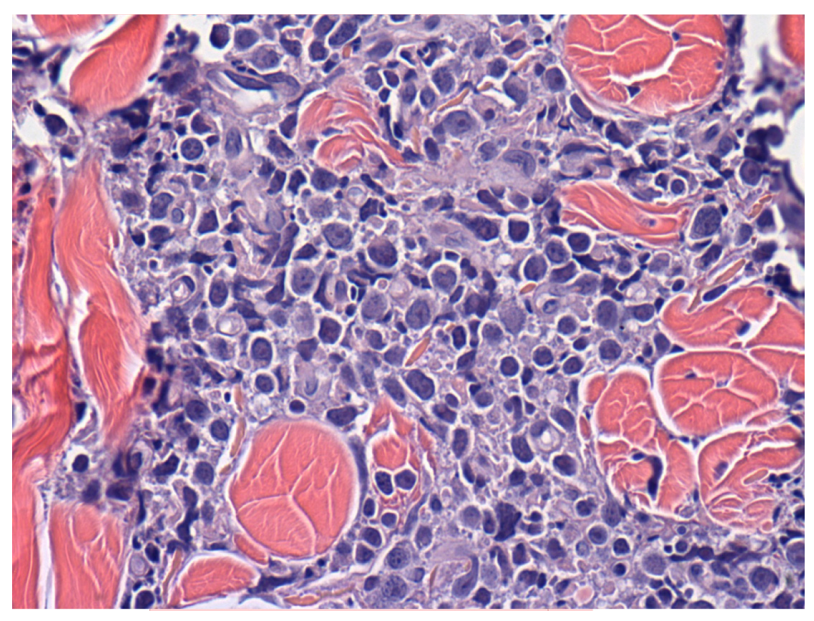

Figure 3 Section of abdominal lesion from skin biopsy.

Notes: Abdomen, magnification $=400 \times$, hematoxylin and eosin staining. The dermal infiltrate is comprised of medium-sized cells with variably irregular nuclear contours, blast-like chromatin, and variably prominent nucleoli.

monocytosis of $1.7 \mathrm{~K} / \mu \mathrm{L}$. PBAM, defined in this paper and others as $>1.0 \mathrm{~K} / \mu \mathrm{L}$, has a higher incidence in patients having both hematologic and nonhematologic malignancies. ${ }^{10,11}$ Recent studies have indicated that this is an adverse prognostic indicator in patients with Hodgkin and non-Hodgkin lymphoma. ${ }^{10,12,13}$ Tadmor et al ${ }^{10}$ studied absolute monocytosis in patients with diffuse large B-cell lymphoma and found that an absolute monocytosis in these patients was an independent prognostic factor associated with decreased overall survival. A recent large multicenter study of 1,191 diffuse large B-cell lymphoma patients also demonstrated that monocytosis (absolute monocyte count $>630 \mathrm{~mm}^{3}$ ) was associated with poor prognosis. ${ }^{14}$ The rationale for this is believed to be from monocytes/macrophages supporting growth and survival
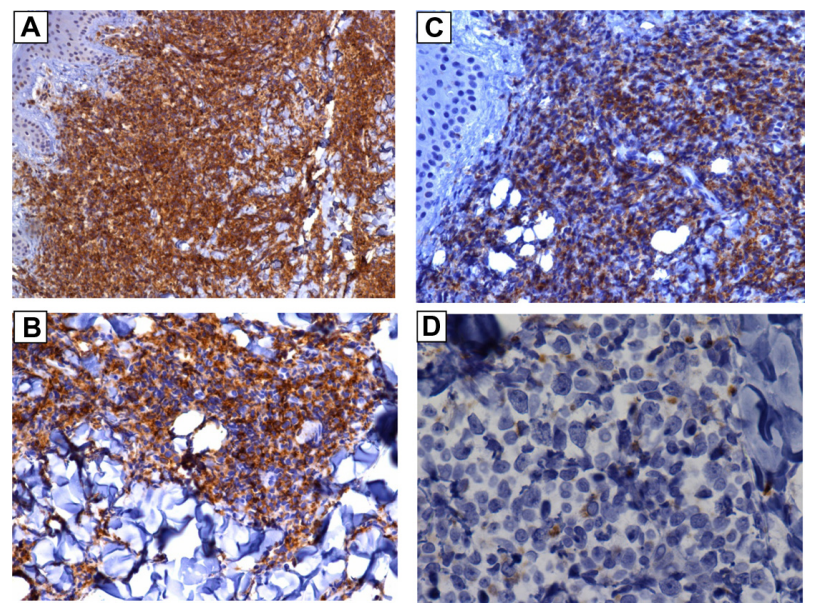

Figure 4 Immunohistochemical panel showing atypical cells positive for CD4, CD43, CD56, and CD68 (dot-like distribution).

Notes: Tumor cells are diffusely positive for (A) CD56, (B) CD43, and (C) CD4. (D) CD68 showed dot positivity. 
of the neoplastic cells in the tumor microenvironment due to immunosuppressive effects. ${ }^{10}$ These tumor-associated macrophages, which are recruited by the neoplastic cells by chemokines, play a role in promoting angiogenesis, immunosuppression, and metastasis. ${ }^{10}$ PBAM has also been associated with worse prognosis in primary myelofibrosis and T-cell leukemia/lymphoma. ${ }^{15,16}$

The association between BPDCN and PBAM has not been emphasized in the literature. There is one case report of a patient having BPDCN with peripheral lymphadenopathy and bone marrow involvement and a "normal differential," even though the monocyte percentage was reported as $24 \%$ with a white blood cell count of $5.1 \mathrm{~K} / \mu \mathrm{L}$, yielding an absolute monocytosis of $1.2 \mathrm{~K} / \mu \mathrm{L} .{ }^{9}$ It appears, then, that the incidence of PBAM and BPDCN is underreported. Of the large case series in the literature, none report the complete blood count differential. ${ }^{1,5}$ It would be intriguing to see what percentage of patients with BPDCN have an absolute monocytosis. This raises many questions, such as the following: Is there, in fact, a higher incidence of absolute monocytosis in patients with BPDCN? If so, is there a difference with respect to the monocyte count between those with and without leukemic dissemination? Can the monocyte count be followed after treatment as a sort of tumor marker? And most importantly, is there any prognostic significance with regard to the monocyte count in patients with BPDCN? Based on the numerous other hematologic malignancies associated with PBAM and worse prognosis, it is possible that such an association exists with BPDCN. However, this can only be answered by examining a large series of patients with sufficient follow-up data.

\section{Disclosure}

The authors report no financial disclosures or relevant relationships. The authors report no other conflicts of interest in this work.

\section{References}

1. Petrella T, Bagot M, Willemze R, et al. Blastic NK-cell lymphomas (agranular CD4+CD56+ hematodermic neoplasms): a review. Am J Clin Pathol. 2005;123(5):662-675.
2. Facchetti F, Jones D, Petrella T. Blastic plasmacytoid dendritic cell neoplasm. In: Swerdlow SH, Campo E, Harris NL, et al, editors. WHO Classification of Tumours of Haematopoietic and Lymphoid Tissues (IARC WHO Classification of Tumours). 4th ed. Lyon, France: IARC Press; 2008:145-147.

3. Adachi M, Maeda K, Takekawa M, et al. High expression of CD56 (N-CAM) in a patient with cutaneous CD4-positive lymphoma. Am J Hematol. 1994;47(4):278-282.

4. Borchiellini D, Ghibaudo N, Mounier N, et al. Blastic plasmacytoid dendritic cell neoplasm: a report of four cases and review of the literature. J Eur Acad Dermatol Venereol. 2013;27(9):1176-1181.

5. Cota C, Vale E, Viana I, et al. Cutaneous manifestations of blastic plasmacytoid dendritic cell neoplasm-morphologic and phenotypic variability in a series of 33 patients. Am J Surg Pathol. 2010;34(1):75-87.

6. Rauh MJ, Rahman F, Good D, et al. Blastic plasmacytoid dendritic cell neoplasm with leukemic presentation, lacking cutaneous involvement: Case series and literature review. Leuk Res. 2012;36(1):81-86.

7. Tsunoda K, Satoh T, Akasaka K, et al. Blastic plasmacytoid dendritic cell neoplasm: report of two cases. J Clin Exp Hematop. 2012;52(1): 23-29.

8. Dalle S, Beylot-Barry M, Bagot M, et al. Blastic plasmacytoid dendritic cell neoplasm: is transplantation the treatment of choice? Br J Dermatol. 2010;162(1):74-79.

9. Male HJ, Davis MB, McGuirk JP, et al. Blastic plasmacytoid dendritic cell neoplasm should be treated with acute leukemia type induction chemotherapy and allogeneic stem cell transplantation in first remission. Int J Hematol. 2010;92(2):398-400.

10. Tadmor T, Fell R, Polliack A, Attias D. Absolute monocytosis at diagnosis correlates with survival in diffuse large B-cell lymphomapossible link with monocytic myeloid-derived suppressor cells. Hematol Oncol. 2013;31(2):65-71.

11. Shoenfeld Y, Tal A, Berliner S, Pinkhas J. Leukocytosis in non hematological malignancies - a possible tumor-associated marker. J Cancer Res Clin Oncol. 1986;111(1):54-58.

12. Wilcox RA, Ristow K, Habermann TM, et al. The absolute monocyte and lymphocyte prognostic score predicts survival and identifies highrisk patients in diffuse large-B-cell lymphoma. Leukemia. 2011;25(9): 1502-1509.

13. Porrata LF, Ristow K, Colgan JP, et al. Peripheral blood lymphocyte/ monocyte ratio at diagnosis and survival in classical Hodgkin's lymphoma. Haematologica. 2012;97(2):262-269.

14. Tadmor T, Bari A, Sacchi S, et al. Monocyte count at diagnosis is a prognostic parameter in diffuse large B-cell lymphoma: results from a large multicenter study involving 1191 patients in the pre- and postrituximab era. Haematologica. 2014;99(1):125-130.

15. Elliot MA, Verstovsek S, Dingli D, et al. Monocytosis is an adverse prognostic factor for survival in younger patients with primary myelofibrosis. Leuk Res. 2007;31(11):1503-1509.

16. Tokioka T, Shimamoto Y, Motoyoshi K, Yamaguchi M. Clinical significance of monocytosis and human monocytic colony-stimulating factor in patients with adult T-cell leukaemia/lymphoma. Haematologia (Budap). 1994;26(1):1-9.
Pathology and Laboratory Medicine International

\section{Publish your work in this journal}

Pathology and Laboratory Medicine International is a peer-reviewed, open access journal focusing on innovative basic research and translational research related to pathology or human disease. The journal includes original research, updates, case reports, reviews and commentaries on current controversies. The Academic Sponsor

\section{Dovepress}

of this journal is the Chinese American Pathology Association (CAPA). The manuscript management system is completely online and includes a very quick and fair peer-review system. Visit http://www.dovepress.com/testimonials.php to read real quotes from published authors. 